\title{
Local energy decay of solutions to the wave equation for nontrapping metrics
}

\author{
Georgi Vodev
}

\begin{abstract}
We prove uniform local energy decay estimates of solutions to the wave equation on unbounded Riemannian manifolds with nontrapping metrics. These estimates are derived from the properties of the resolvent at high frequency. Applications to a class of asymptotically Euclidean manifolds as well as to perturbations by non-negative long-range potentials are given.
\end{abstract}

\section{Introduction and statement of results}

Let $(M, g)$ be an $n$-dimensional unbounded connected Riemannian manifold with a Riemannian metric $g$ of class $C^{\infty}(\bar{M})$ and a compact $C^{\infty}$-smooth boundary $\partial M$ (which may be empty). We suppose that $M$ is of the form $M=X_{0} \cup X$, where $X_{0}$ is a compact connected Riemannian manifold with a metric $\left.g\right|_{X_{0}}$ of class $C^{\infty}\left(\bar{X}_{0}\right)$ with a compact boundary $\partial X_{0}=\partial M \cup \partial X, \partial M \cap \partial X=\emptyset, X=\left[r_{0},+\infty\right) \times S$, $r_{0} \gg 1$, with metric $\left.g\right|_{X}:=d r^{2}+\sigma(r)$. Here $(S, \sigma(r))$ is an $(n-1)$-dimensional compact Riemannian manifold without boundary equipped with a family of Riemannian metrics $\sigma(r)$ depending smoothly on $r$ which can be written in any local coordinates $\theta \in S$ in the form

$$
\sigma(r)=\sum_{i, j=1}^{n-1} g_{i j}(r, \theta) d \theta_{i} d \theta_{j}, \quad g_{i j} \in C^{\infty}(X)
$$

Let $X_{r}=[r,+\infty) \times S$. Clearly, $\partial X_{r}$ can be identified with the Riemannian manifold $(S, \sigma(r))$ with the Laplace-Beltrami operator $\Delta_{\partial X_{r}}$ written as

$$
\Delta_{\partial X_{r}}=-\frac{1}{p} \sum_{i, j=1}^{n-1} \partial_{\theta_{i}}\left(p g^{i j} \partial_{\theta_{j}}\right)
$$

where $\left(g^{i j}\right)$ is the inverse matrix to $\left(g_{i j}\right)$ and $p=\left(\operatorname{det}\left(g_{i j}\right)\right)^{1 / 2}=\left(\operatorname{det}\left(g^{i j}\right)\right)^{-1 / 2}$. Let $\Delta_{g}$ denote the (positive) Laplace-Beltrami operator on $(M, g)$ and let $\nabla_{g}$ be the 
corresponding gradient. We have

$$
\Delta_{X}:=\left.\Delta_{g}\right|_{X}=-\frac{1}{p} \partial_{r}\left(p \partial_{T}\right)+\Delta_{\partial X_{r}}=-\partial_{r}^{2}-\frac{p^{\prime}}{p} \partial_{r}+\Delta_{\partial X_{r}},
$$

where $p^{\prime}=\partial p / \partial r$. We have the identity

$$
\Delta_{X}^{\sharp}:=p^{1 / 2} \Delta_{X} p^{-1 / 2}=-\partial_{r}^{2}+\Lambda_{r}+q(r, \theta),
$$

where

$$
\Lambda_{r}=-\sum_{i, j=1}^{n-1} \partial_{\theta_{i}}\left(g^{i j} \partial_{\theta_{j}}\right)
$$

and $q$ is an effective potential given by

$$
q(r, \theta)=(2 p)^{-2}\left(\frac{\partial p}{\partial r}\right)^{2}+(2 p)^{-2} \sum_{i, j=1}^{n-1} \frac{\partial p}{\partial \theta_{i}} \frac{\partial p}{\partial \theta_{j}} g^{i j}+\frac{1}{2} p \Delta_{X}\left(p^{-1}\right)
$$

We suppose that $q=q_{1}+q_{2}$, where $q_{1}$ and $q_{2}$ are real-valued functions satisfying

$$
\left|q_{1}(r, \theta)\right| \leq C, \quad \frac{\partial q_{1}}{\partial r}(r, \theta) \leq C r^{-1-\delta_{0}} \quad \text { and } \quad\left|q_{2}(r, \theta)\right| \leq C r^{-1-\delta_{0}}
$$

with constants $C, \delta_{0}>0$. Let

$$
h(r, \theta, \xi)=\sum_{i, j=1}^{n-1} g^{i j}(r, \theta) \xi_{i} \xi_{j}, \quad(\theta, \xi) \in T^{*} S
$$

We also suppose that

$$
-\frac{\partial h}{\partial r}(r, \theta, \xi) \geq \frac{C}{r} h(r, \theta, \xi) \text { for }(\theta, \xi) \in T^{*} S,
$$

with a constant $C>0$.

Denote by $G$ the selfadjoint realization of $\Delta_{g}$ on the Hilbert space

$$
H=L^{2}\left(M, d \mathrm{Vol}_{g}\right)
$$

with Dirichlet or Neumann boundary conditions, $B u=0$, on $\partial M$. Let $\chi \in C^{\infty}(\bar{M})$, $\chi=1$ on $X_{0}, \chi=0$ in $X_{r_{0}+1}$. Let $\mathcal{G}:=(G+1)^{1 / 2}$. We make the following assumption:

$$
\begin{aligned}
& \text { there exist constants } T, \sigma>0 \text { so that the operators } \\
& \mathcal{G}^{\sigma_{1}} \chi \cos (T \sqrt{G}) \chi \mathcal{G}^{\sigma_{2}} \text { and } \mathcal{G}^{\sigma_{1}} \chi(\sin (T \sqrt{G}) / \sqrt{G}) \chi \mathcal{G}^{\sigma_{2}+1}
\end{aligned}
$$$$
\text { belong to } \mathcal{L}(H) \text { for all } \sigma_{1}, \sigma_{2} \in \mathbf{R} \text { such that } \sigma_{1}+\sigma_{2}=\sigma \text {. }
$$ 
The metric $g$ will be said nontrapping if there exists a constant $T_{0}>0$ such that for every generalized geodesics $\gamma$ (see [3] and [4] for the definition), with $\gamma(0) \in$ $M \backslash X_{T_{0}+1 / 2}$, there is $0<t=t_{\gamma} \leq T_{0}$ so that $\gamma(t) \in X_{r_{0}+1}$. For such a metric, it follows from the result of Melrose-Sjöstrand [3], [4] on propagation of $C^{\infty}$ singularities that the distribution kernels of the operators $\chi \cos \left(T_{0} \sqrt{G}\right) \chi$ and $\chi\left(\sin \left(T_{0} \sqrt{G}\right) / \sqrt{G}\right) \chi$ are of class $C^{\infty}(\bar{M} \times \bar{M})$ (this is known as the generalized Huyghens principle). Therefore, (1.4) is fulfilled for nontrapping metrics.

Given a real $s$, choose a real-valued function $\chi_{s} \in C^{\infty}(\bar{M}), \chi_{s}=1$ on $M \backslash X_{r_{0}+1 / 2}$, $\left.\chi_{s}\right|_{X}$ depending only on $r, \chi_{s}=r^{-s}$ on $X_{r_{0}+1}, \chi_{s} \chi_{-s} \equiv 1$. Denote by $G_{0}$ the Dirichlet selfadjoint realization of $\Delta_{X}$ on the Hilbert space $H_{0}=L^{2}\left(X, d \mathrm{Vol}_{g}\right)$.

Our first result is the following theorem.

Theorem 1.1. Assume (1.2) and (1.3) fulfilled. Then, for every $s>\frac{1}{2}$, there exist constants $C_{0}, C>0$ such that for $z \geq C_{0}$ and $0<\varepsilon \leq 1$, we have (with $j=0,1$ )

$$
\left\|r^{-s} \nabla_{g}^{j}\left(G_{0}-z \pm i \varepsilon\right)^{-1} r^{-s}\right\|_{\mathcal{L}\left(H_{0}\right)} \leq C z^{(j-1) / 2} .
$$

Moreover, if (1.4) holds, we have

$$
\left\|\chi_{s} \nabla_{g}^{j}(G-z \pm i \varepsilon)^{-1} \chi_{s}\right\|_{\mathcal{L}(H)} \leq C z^{(j-1) / 2}
$$

We will use this theorem to study the local energy of the solutions of the following mixed problem

$$
\begin{cases}\left(\partial_{t}^{2}+\Delta_{g}\right) u(t, x)=0 & \text { in } \mathbf{R} \times M, \\ B u(t, x)=0 & \text { on } \mathbf{R} \times \partial M, \\ u(0, x)=\varphi(G) f_{1}(x), \partial_{t} u(0, x)=\varphi(G) f_{2}(x), & x \in M,\end{cases}
$$

where $\varphi \in C^{\infty}(\mathbf{R}), \varphi=0$ in a neighbourhood of the interval $\left(-\infty, A_{0}\right], \varphi=1$ outside a larger neighbourhood, and $A_{0}=\max \left\{C_{0}, C_{0}^{\prime}\right\}, C_{0}$ being as in Theorem 1.1 and $C_{0}^{\prime}$ being as in (1.9) and (1.10) below. Recall that the solutions to $(1.7)$ can be expressed by the formula

$$
u=\cos (t \sqrt{G}) \varphi(G) f_{1}+\frac{\sin (t \sqrt{G})}{\sqrt{G}} \varphi(G) f_{2} .
$$

Given $s>0$ and a function $\chi \in C^{\infty}(\bar{M}), \chi=1$ in $X_{0}, \chi=0$ outside some compact set, let

$$
\begin{aligned}
E_{s}(t) & =\int_{M}\left(\left|\partial_{t} u(t, x)\right|^{2}+\left|\nabla_{g} u(t, x)\right|^{2}\right) \chi_{2 s} d \mathrm{Vol}_{g} \\
E_{\mathrm{loc}}(t) & =\int_{M}\left(\left|\partial_{t} u(t, x)\right|^{2}+\left|\nabla_{g} u(t, x)\right|^{2}\right) \chi d \mathrm{Vol}_{g} \\
\widetilde{E}(0) & =\int_{M}\left(\left|f_{2}\right|^{2}+\left|\nabla_{g} f_{1}\right|^{2}+\left|f_{1}\right|^{2}\right) d \mathrm{Vol}_{g} \\
\widetilde{E}_{-s}(0) & =\int_{M}\left(\left|f_{2}\right|^{2}+\left|\nabla_{g} f_{1}\right|^{2}\right) \chi_{-2 s} d \operatorname{Vol}_{g}+\int_{M}\left|f_{1}\right|^{2} \chi_{-2 s+2} d \mathrm{Vol}_{g}
\end{aligned}
$$


To get uniform local energy decay estimates of the solutions to (1.7) we need to impose additional conditions on the behaviour of the resolvent of the operator $G_{0}$. We suppose that there exist $s>\frac{1}{2}, C_{0}^{\prime}>0$ and an integer $m \geq 0$ so that for $z \geq C_{0}^{\prime}$, $0<\varepsilon \leq 1$, the following estimates hold (with $j=0,1$ ):

$$
\begin{aligned}
& \left\|r^{-s} \nabla_{g}^{j}\left(G_{0}-z \pm i \varepsilon\right)^{-k} r^{-s}\right\|_{\mathcal{L}\left(H_{0}\right)} \leq C z^{(j-k) / 2}, \quad k=1, \ldots, m+1, \\
& \left\|r^{-s} \nabla_{g}^{j}\left(G_{0}-z \pm i \varepsilon z^{1 / 2}\right)^{-m-2} r^{-s}\right\|_{\mathcal{L}\left(H_{0}\right)} \leq C z^{(j-m-2) / 2} \varepsilon^{-1+\mu},
\end{aligned}
$$

with constants $C>0$ and $0<\mu \leq 1$ independent of $z$ and $\varepsilon$.

Our main result is the following theorem.

Theorem 1.2. Assume (1.2), (1.3), (1.4), (1.9) and (1.10) fulfilled. Then, we have, for $t \gg 1$,

$$
E_{s-1 / 2}(t) \leq O\left(t^{-2 m-2 \mu}\right) \widetilde{E}_{-s}(0) .
$$

In particular, if $f_{1}$ and $f_{2}$ are of compact support, we have

$$
E_{\mathrm{loc}}(t) \leq O\left(t^{-2 m-2 \mu}\right) \widetilde{E}(0) .
$$

Remark 1. If (1.9) holds for every integer $k \geq 1$ with $s=s_{k}>\frac{1}{2}$ and $C=C_{k}>0$, then (1.12) holds with $O_{N}\left(t^{-N}\right)$ for every $N \gg 1$, in place of $O\left(t^{-2 m-2 \mu}\right)$.

The key point in the proof of Theorem 1.2 is the following result.

Proposition 1.3. The estimates (1.5), (1.6), (1.9) and (1.10) imply the following estimates (with $j=0,1$ )

$$
\begin{aligned}
& \left\|\chi_{s} \nabla_{g}^{j}(G-z \pm i \varepsilon)^{-k} \chi_{s}\right\|_{\mathcal{L}(H)} \leq C z^{(j-k) / 2}, \quad k=1, \ldots, m+1, \\
& \left\|\chi_{s} \nabla_{g}^{j}\left(G-z \pm i \varepsilon z^{1 / 2}\right)^{-m-2} \chi_{s}\right\|_{\mathcal{L}(H) \leq C z^{(j-m-2) / 2} \varepsilon^{-1+\mu}}
\end{aligned}
$$

for $z \geq A_{0}, 0<\varepsilon \leq 1$, with $m$ and $0<\mu \leq 1$ being the same as in (1.9) and (1.10), and a new constant $C>0$ independent of $z$ and $\varepsilon$.

As an application of the above theorem we will get uniform local energy decay of the solutions to (1.7) for a class of asymptotically Euclidean manifolds. To describe this class, let

$$
q^{b}:=\frac{1}{r} \frac{\partial\left(r^{2} q\right)}{\partial r} \quad \text { and } \quad \Lambda_{r}^{b}:=-\frac{1}{r} \sum_{i, j} \partial_{\theta_{i}}\left(\frac{\partial\left(r^{2} g^{i j}\right)}{\partial r} \partial_{\theta_{j}}\right)
$$

Denote by $G_{0}^{\sharp}$ the Dirichlet selfadjoint realization of the operator $\Delta_{X}^{\sharp}$ on the Hilbert space $H_{0}^{\sharp}=L^{2}(X, d r d \theta)$. We make the assumptions

$$
\begin{aligned}
\left|q^{b}(r, \theta)\right| & \leq C r^{-\delta}, \\
r^{\delta} \Lambda_{r}^{b}\left(G_{0}^{\sharp}-i\right)^{-1} & \in \mathcal{L}\left(H_{0}^{\sharp}\right),
\end{aligned}
$$

for some constants $C>0$ and $0<\delta \leq 1$. We have the following results. 
Proposition 1.4. Assume (1.2), (1.3), (1.15) and (1.16) fulfilled. Then, (1.9) and (1.10) hold with $m=0$ and $s=1+\frac{1}{2} \delta$ for every $0<\mu<\delta$.

Corollary 1.5. Assume (1.2), (1.3), (1.4), (1.15) and (1.16) fulfilled. Then, we have, for $t \gg 1$,

$$
E_{(1+\delta) / 2}(t) \leq O_{\varepsilon}\left(t^{-2 \delta+\varepsilon}\right) \tilde{E}_{-(1+\delta / 2)}(0) \quad \text { for every } 0<\varepsilon \ll 1 .
$$

In particular, if $f_{1}$ and $f_{2}$ are of compact support, we have

$$
E_{\mathrm{loc}}(t) \leq O_{\varepsilon}\left(t^{-2 \delta+\varepsilon}\right) \widetilde{E}(0) \quad \text { for every } 0<\varepsilon \ll 1 .
$$

Remark 2. It is worth noticing that the above results still hold for the selfadjoint realization of $\Delta_{g}+V(x)$, where $V$ is a real-valued potential, $V(x) \geq 0$, provided the assumptions (1.2) and (1.15) are satisfied with $q$ replaced by $q+\left.V\right|_{X}$.

It is easy to see that the assumptions (1.2), (1.3), (1.15) and (1.16) are fulfilled for long-range perturbations of the Euclidean metric on $\mathbf{R}^{n}, n \geq 2$. More precisely, let $\mathcal{O} \subset \mathbf{R}^{n}$ be a bounded domain with a $C^{\infty}$-smooth boundary and connected complement $\Omega=\mathbf{R}^{n} \backslash \mathcal{O}$. Let $g$ be a Riemannian metric in $\Omega$ of the form

$$
g=\sum_{i, j=1}^{n} g_{i j}(x) d x_{i} d x_{j}, \quad g_{i j}(x) \in C^{\infty}(\bar{\Omega}),
$$

satisfying

$$
\left|\partial_{x}^{\alpha}\left(g_{i j}(x)-\delta_{i j}\right)\right| \leq C_{\alpha}\langle x\rangle^{-\gamma_{0}-|\alpha|}
$$

for every multi-index $\alpha$, with constants $C_{\alpha}, \gamma_{0}>0$, where $\langle x\rangle:=\left(1+|x|^{2}\right)^{1 / 2}$ and $\delta_{i j}$ denotes the Kronecker symbol. It follows from (1.19) that outside a sufficiently large compact set there exists a global smooth change of variables, $(r, \theta)=(r(x), \theta(x))$, $r \in\left[r_{0},+\infty\right), r_{0} \gg 1, \theta \in S=\left\{y \in \mathbf{R}^{n}:|y|=1\right\}$, which transforms the metric $g$ to the form $d r^{2}+\sigma(r)$. Therefore, $(\Omega, g)$ is isometric to a Riemannian manifold of the class described above, and (1.17) and $(1.18\}$ hold with $\delta=\min \left\{1, \gamma_{0}\right\}$, provided the metric $g$ is nontrapping.

In the case when $g_{i j}=\delta_{i j}$ for $|x| \geq \varrho_{0}$ with some $\varrho_{0} \gg 1$, and the metric $g$ is nontrapping, a better estimate than (1.18) is known to hold true with $\varphi \equiv 1$. In this case, Vainberg [5], [6] showed that the generalized Huyghens principle implies (1.18) with a rate of decay $O\left(e^{-c t}\right), c>0$, if $n \geq 3$ is odd, and $O\left(t^{-2 n}\right)$ if $n \geq 4$ is even. The fact that the metric coincides with the Euclidean one outside some compact set plays an important role in Vainberg's method. In particular, this implies that 
the cutoff resolvent extends analytically to some strip through the real axis. This approach, however, does not work anymore in the setting described above, and it does not allow one to get estimates like (1.11) and (1.17).

To prove Theorem 1.1 we make use of some ideas developed in [1] and [8], where uniform high frequency resolvent estimates have been obtained without assuming (1.4). The assumption (1.4), however, allows to get much better estimates than those proved in the above papers. We use it to prove a priori estimates for the stationary problem on some large compact set (see Proposition 2.1 below). On the other hand, outside another large compact set we have nice a priori estimates due to the assumptions (1.2) and (1.3) (see Proposition 2.3 below, which is proved in [1]). Then, we paste together both a priori estimates in precisely the same way as in [1] in order to get (1.6). Note that a semi-classical analogue of (1.6) is proved in [7] for a class of asymptotically Euclidean manifolds and nontrapping metrics. Proposition 1.4 is proved using some ideas from [2], where a similar result has been proved.

\section{High frequency resolvent estimates}

In this section we will prove Theorem 1.1 as well as Propositions 1.3 and 1.4. In what follows, given any domain $M_{0} \subset M$, the Sobolev space $H^{1}\left(M_{0}, d \mathrm{Vol}_{g}\right)$ will be equipped with the semi-classical norm defined by

$$
\|u\|_{H^{1}\left(M_{0}, d \mathrm{Vol}_{g}\right)}^{2}:=\|u\|_{L^{2}\left(M_{0}, d \mathrm{Vol}_{g}\right)}^{2}+\left\|\lambda^{-1} \nabla_{g} u\right\|_{L^{2}\left(M_{0}, d \mathrm{Vol}_{g}\right)}^{2},
$$

where $\lambda \gg 1$. We begin with the proof of Theorem 1.1. In fact, the estimate (1.5) is proved in [8], Theorem 2.1, and we therefore omit the proof. To prove (1.6) we need the following result.

Proposition 2.1. Assume (1.4) fulfilled. Then, given any $u \in D(G)$, the following estimate holds:

$$
\begin{aligned}
\|u\|_{H^{1}\left(M \backslash X_{r_{0}+1}, d \mathrm{Vol}_{g}\right) \leq} & \frac{C}{\lambda}\left\|\left(\Delta_{g}-\lambda^{2}+i \varepsilon\right) u\right\|_{L^{2}\left(M \backslash X_{r_{0}+1}, d \mathrm{Vol}_{g}\right)} \\
& +C\|u\|_{H^{1}\left(X_{r_{0}+1 / 2} \backslash X_{r_{0}+1}, d \mathrm{Vol}_{g}\right)}
\end{aligned}
$$

for $\lambda \geq \lambda_{0}$ and $0<\varepsilon \leq 1$, with constants $C, \lambda_{0}>0$ independent of $\lambda$ and $\varepsilon$.

Proof. Choose a function $\eta \in C^{\infty}(\bar{M}), \eta=1$ in $M \backslash X_{r_{0}+1 / 2}, \eta=0$ in $X_{r_{0}+1}$, and set $w=\eta u \in D(G)$. Clearly, (2.1) follows from the estimate

$$
\|w\|_{H^{1}\left(M \backslash X_{r_{0}+1}, d \mathrm{Vol}_{g}\right)} \leq \frac{C}{\lambda}\left\|\left(\Delta_{g}-\lambda^{2}+i \varepsilon\right) w\right\|_{L^{2}\left(M \backslash X_{r_{0}+1}, d \mathrm{Vol}_{g}\right)}
$$

for $\lambda \geq \lambda_{0}$ and $0<\varepsilon \leq 1$, with constants $C, \lambda_{0}>0$ independent of $\lambda$ and $\varepsilon$. We will derive (2.2) from the following a priori estimate. 
Proposition 2.2. Let $U(t, x)=0$ in $\mathbf{R} \times X_{r_{0}+1}$ satisfy the equation

$$
\begin{cases}\left(\partial_{t}^{2}+\Delta_{g}\right) U(t, x)=V(t, x) & \text { in } \mathbf{R} \times M, \\ B U(t, x)=0 & \text { on } \mathbf{R} \times \partial M\end{cases}
$$

Under the assumption (1.4), there exists a constant $C>0$ so that the following inequality holds:

$$
\begin{aligned}
\left\|\partial_{t} U(T, \cdot)\right\|+\left\|\nabla_{g} U(T, \cdot)\right\| \leq & C\left\|\mathcal{G}^{1-\sigma} U(0, \cdot)\right\| \\
& +C\left\|\mathcal{G}^{-\sigma} \partial_{t} U(0, \cdot)\right\|+C \int_{0}^{T}\|V(t, \cdot)\| d t,
\end{aligned}
$$

where $T, \sigma>0$ are as in (1.4). Here and below $\|\cdot\|$ denotes the norm in the space $L^{2}\left(M, d \mathrm{Vol}_{g}\right)$.

Proof. By Duhamel's formula we have

$$
\begin{aligned}
U(t, \cdot)= & \cos (t \sqrt{G}) U(0, \cdot)+\frac{\sin (t \sqrt{G})}{\sqrt{G}} \partial_{t} U(0, \cdot) \\
& +\int_{0}^{t} \frac{\sin ((t-\tau) \sqrt{G})}{\sqrt{G}} V(\tau, \cdot) d \tau .
\end{aligned}
$$

Let $\chi \in C^{\infty}(\bar{M}), \chi=1$ on $\operatorname{supp} U, \chi=0$ outside a small neighbourhood of $\operatorname{supp} U$. In view of (2.5) we can write

$$
\begin{aligned}
\partial_{t} U(t, \cdot)= & -G \chi \frac{\sin (t \sqrt{G})}{\sqrt{G}} \chi \mathcal{G}^{\sigma-1} \mathcal{G}^{1-\sigma} U(0, \cdot) \\
& +[G, \chi] \frac{\sin (t \sqrt{G})}{\sqrt{G}} \chi \mathcal{G}^{\sigma-1} \mathcal{G}^{1-\sigma} U(0, \cdot) \\
& +\chi \cos (t \sqrt{G}) \chi \mathcal{G}^{\sigma} \mathcal{G}^{-\sigma} \partial_{t} U(0, \cdot) \\
& +\int_{0}^{t} \chi \cos ((t-\tau) \sqrt{G}) \chi V(\tau, \cdot) d \tau \\
\nabla_{g} U(t, \cdot)= & \nabla_{g} \chi \cos (t \sqrt{G}) \chi \mathcal{G}^{\sigma-1} \mathcal{G}^{1-\sigma} U(0, \cdot) \\
& +\nabla_{g} \chi \frac{\sin (t \sqrt{G})}{\sqrt{G}} \chi \mathcal{G}^{\sigma} \mathcal{G}^{-\sigma} \partial_{t} U(0, \cdot) \\
& +\int_{0}^{t} \chi \nabla_{g} \frac{\sin ((t-\tau) \sqrt{G})}{\sqrt{G}} \chi V(\tau, \cdot) d \tau \\
& +\int_{0}^{t}\left[\nabla_{g}, \chi\right] \frac{\sin ((t-\tau) \sqrt{G})}{\sqrt{G}} \chi V(\tau, \cdot) d \tau .
\end{aligned}
$$


In view of Green's formula we have

$$
\left\|\nabla_{g} f\right\|^{2}=\left\langle\Delta_{g} f, f\right\rangle=\langle G f, f\rangle=\|\sqrt{G} f\|^{2} \text { for } f \in D(G) .
$$

It is easy to see that (2.4) follows from (2.6)-(2.8) together with (1.4).

We are going to apply (2.4) with

$$
U(t, x)=e^{i t \lambda_{1}} w(x) \text { and } \quad V(t, x)=e^{i t \lambda_{1}}\left(\Delta_{g}-\lambda^{2}+i \varepsilon\right) w,
$$

where $\lambda_{1} \in \mathbf{C}$ is such that $\lambda_{1}^{2}=\lambda^{2}-i \varepsilon$ and $\operatorname{Re} \lambda_{1}>0$. We obtain

$$
\lambda\|w\|+\left\|\nabla_{g} w\right\| \leq C\left\|\mathcal{G}^{1-\sigma} w\right\|+C \lambda\left\|\mathcal{G}^{-\sigma} w\right\|+C\left\|\left(\Delta_{g}-\lambda^{2}+i \varepsilon\right) w\right\| .
$$

Without loss of generality we may suppose that $\sigma \leq 1$. We are going to show that, for $\lambda \geq 1$,

$$
\begin{aligned}
\left\|\mathcal{G}^{1-\sigma} w\right\| & \leq C \lambda^{1-\sigma}\|w\|+C\left\|\left(\Delta_{g}-\lambda^{2}+i \varepsilon\right) w\right\|, \\
\left\|\mathcal{G}^{-\sigma} w\right\| & \leq C \lambda^{-\sigma /(2-\sigma)}\|w\|+\frac{C}{\lambda}\left\|\left(\Delta_{g}-\lambda^{2}+i \varepsilon\right) w\right\| .
\end{aligned}
$$

Given a parameter $A>1$, denote by $\psi$ the characteristic function of the interval $(-\infty, A]$. We have

$$
\begin{aligned}
\left\|\mathcal{G}^{1-\sigma} w\right\| & \leq\left\|\mathcal{G}^{1-\sigma} \psi(\mathcal{G}) w\right\|+\left\|\mathcal{G}^{1-\sigma}(1-\psi(\mathcal{G})) w\right\| \leq A^{1-\sigma}\|w\|+A^{-1-\sigma}\|(G+1) w\| \\
& \leq A^{1-\sigma}\|w\|+A^{-1-\sigma} O\left(\lambda^{2}\right)\|w\|+O(1)\left\|\left(\Delta_{g}-\lambda^{2}+i \varepsilon\right) w\right\|
\end{aligned}
$$

for all $A>1$. Choosing $A=\lambda$ we obtain (2.10). In the same way, we have

$$
\left\|\mathcal{G}^{-\sigma} w\right\| \leq\left\|\mathcal{G}^{-\sigma}(1-\psi(\mathcal{G})) w\right\|+\left\|\mathcal{G}^{-\sigma} \psi(\mathcal{G}) w\right\| \leq A^{-\sigma}\|w\|+A^{2-\sigma}\left\|(G+1)^{-1} w\right\| .
$$

On the other hand,

$$
\begin{aligned}
\left\|(G+1)^{-1} w\right\| & =\frac{1}{\lambda^{2}}\left\|(G+1)^{-1}\left(\Delta_{g}-\lambda^{2}+i \varepsilon\right) w+(1-i \varepsilon)(G+1)^{-1} w-w\right\| \\
& \leq O\left(\lambda^{-2}\right)\left(\left\|\left(\Delta_{g}-\lambda^{2}+i \varepsilon\right) w\right\|+\|w\|\right) .
\end{aligned}
$$

Hence,

$$
\left\|\mathcal{G}^{-\sigma} w\right\| \leq A^{-\sigma}\|w\|+A^{2-\sigma} O\left(\lambda^{-2}\right)\|w\|+A^{2-\sigma} O\left(\lambda^{-2}\right)\left\|\left(\Delta_{g}-\lambda^{2}+i \varepsilon\right) w\right\|
$$

for all $A>1$. Choosing $A=\lambda^{1 /(2-\sigma)}$ we obtain (2.11).

Clearly, (2.2) follows from (2.9), (2.10) and (2.11) by taking $\lambda$ large enough. The proof of Proposition 2.1 is therefore complete. 
The following proposition is proved in [1] (see Proposition 2.4) in the case when $q_{2} \equiv 0$. Since the general case is treated in precisely the same way, we omit the proof.

Proposition 2.3. Let $u \in H^{2}\left(X_{a}, d \mathrm{Vol}_{g}\right), a>r_{0}$, be such that

$$
r^{s}\left(\Delta_{g}-\lambda^{2}+i \varepsilon\right) u \in L^{2}\left(X_{a}, d \operatorname{Vol}_{g}\right)
$$

for $\frac{1}{2}<s \leq \frac{1}{2}\left(1+\delta_{0}\right), 0<\varepsilon \leq 1$. Then, for every $0<\gamma \ll 1$ there exist constants $C_{1}, C_{2}$, $\lambda_{0}>0$ (which may depend on $\gamma$ but are independent of $\lambda$ and $\varepsilon$ ) so that for $\lambda \geq \lambda_{0}$ and all $a_{1}>a$, we have

$$
\begin{aligned}
\left\|r^{-s} u\right\|_{H^{1}\left(X_{a_{1}}, d \mathrm{Vol}_{g}\right)}^{2} \leq & \frac{C_{1}}{\lambda^{2}}\left\|r^{s}\left(\Delta_{g}-\lambda^{2}+i \varepsilon\right) u\right\|_{L^{2}\left(X_{a}, d \operatorname{Vol}_{g}\right)}^{2} \\
& -\frac{C_{2}}{\lambda} \operatorname{Im}\left\langle\partial_{r} u, u\right\rangle_{L^{2}\left(\partial X_{a}\right)}+\gamma\|u\|_{H^{1}\left(X_{a} \backslash X_{a_{1}}, d \operatorname{Vol}_{g}\right)}^{2} .
\end{aligned}
$$

By Green's formula we have

$$
\begin{aligned}
-\operatorname{Im}\left\langle\partial_{r} u, u\right\rangle_{L^{2}\left(\partial X_{a}\right)} & =-\operatorname{Im}\left\langle\left(\Delta_{g}-\lambda^{2}+i \varepsilon\right) u, u\right\rangle_{L^{2}\left(M \backslash X_{a}, d \mathrm{Vol}_{g}\right)}-\varepsilon\|u\|_{L^{2}\left(M \backslash X_{a}, d \mathrm{Vol}_{g}\right)}^{2} \\
& \leq C \gamma_{1} \lambda\left\|\chi_{s} u\right\|_{L^{2}\left(M, d \mathrm{Vol}_{g}\right)}^{2}+\frac{C}{\gamma_{1} \lambda}\left\|\chi_{-s}\left(\Delta_{g}-\lambda^{2}+i \varepsilon\right) u\right\|_{L^{2}\left(M, d \mathrm{Vol}_{g}\right)}^{2}
\end{aligned}
$$

for all $\gamma_{1}>0$. Choose $a=r_{0}+\frac{1}{4}$ and $a_{1}=r_{0}+\frac{1}{3}$. Combining (2.1), (2.12) and (2.13), and choosing the parameters $\gamma$ and $\gamma_{1}$ small enough easily leads to the estimate

$$
\left\|\chi_{s} u\right\|_{H^{1}\left(M, d \mathrm{Vol}_{g}\right)} \leq \frac{C}{\lambda}\left\|\chi_{-s}\left(\Delta_{g}-\lambda^{2}+i \varepsilon\right) u\right\|_{L^{2}\left(M, d \mathrm{Vol}_{g}\right)}, \quad u \in D(G)
$$

for $\lambda \geq \lambda_{0}$ with constants $C, \lambda_{0}>0$ independent of $\lambda$ and $\varepsilon$. Clearly, (2.14) implies (1.6) with $z=\lambda^{2}$. Thus we have completed the proof of Theorem 1.1.

Proof of Proposition 1.3. Choose a function $\varrho \in C^{\infty}(\bar{M}), \varrho=1$ on $M \backslash X_{r_{0}+1}$, $\varrho=0$ on $X_{r_{0}+2}$. We have, for all $\varepsilon>0$,

$$
\begin{aligned}
(G-z \pm i \varepsilon)^{-2}= & (G-z \pm i \varepsilon)^{-1} \varrho(2-\varrho)(G-z \pm i \varepsilon)^{-1} \\
& +(G-z \pm i \varepsilon)^{-1}(1-\varrho)^{2}(G-z \pm i \varepsilon)^{-1} \\
= & (G-z \pm i \varepsilon)^{-1} \varrho(2-\varrho)(G-z \pm i \varepsilon)^{-1} \\
& +\left((G-z \pm i \varepsilon)^{-1}\left[\Delta_{g}, \varrho\right]+1-\varrho\right)\left(G_{0}-z \pm i \varepsilon\right)^{-2} \\
& \times\left(1-\varrho+\left[\varrho, \Delta_{g}\right](G-z \pm i \varepsilon)^{-1}\right) .
\end{aligned}
$$


Differentiating (2.15), $k-1$ times with respect to $z$, we get the identity

$$
\begin{aligned}
(G-z \pm i \varepsilon)^{-k-1}= & \sum_{\nu=1}^{k} c_{k, \nu}(G-z \pm i \varepsilon)^{-\nu} \varrho(2-\varrho)(G-z \pm i \varepsilon)^{-k-1+\nu} \\
& +\sum_{\substack{\nu_{1}+\nu_{2}+\nu_{3}=k+3 \\
\nu_{1} \geq 1 \\
\nu_{2} \geq 2 \\
\nu_{3} \geq 1}} \alpha_{k, \nu_{1}, \nu_{2}, \nu_{3}}\left((G-z \pm i \varepsilon)^{-\nu_{1}}\left[\Delta_{g}, \varrho\right]+\beta_{\nu_{1}}(1-\varrho)\right) \\
& \times\left(G_{0}-z \pm i \varepsilon\right)^{-\nu_{2}}\left(\beta_{\nu_{3}}(1-\varrho)+\left[\varrho, \Delta_{g}\right](G-z \pm i \varepsilon)^{-\nu_{3}}\right),
\end{aligned}
$$

where $c_{k, \nu}, \alpha_{k, \nu_{1}, \nu_{2}, \nu_{3}}$ and $\beta_{\nu}$ are real numbers, $\beta_{\nu}=1$ if $\nu=1$ and $\beta_{\nu}=0$ if $\nu>1$. It follows from (2.16) that if (1.13) holds for $k=1, \ldots, K$ with some integer $1 \leq K \leq m$, then this together with (1.9) imply that it holds for $k=K+1$ as well. Moreover, applying (2.16) with $k=m+1$ and $\varepsilon$ replaced by $\varepsilon z^{1 / 2}$, it is easy to see that (1.14) follows from (1.9), (1.10) and (1.13).

Proof of Proposition 1.4. We will first prove (1.10) for $j=0$ with $m=0, s=$ $1+\frac{1}{2} \delta$. Since the operators $G_{0}$ and $G_{0}^{\sharp}$ are unitarily equivalent, it suffices to prove it with $G_{0}$ and $H_{0}$ replaced by $G_{0}^{\sharp}$ and $H_{0}^{\sharp}$, respectively. Set $\varepsilon_{1}:=\varepsilon z^{1 / 2}, 0<\varepsilon \leq 1$, and

$$
\mathcal{A}:=2 \Delta_{g}^{\sharp}+\left[r \partial_{r}, \Delta_{g}^{\sharp}\right]=q^{b}+\Lambda_{r}^{b} .
$$

For $s>1$ we have the identity

$$
\begin{aligned}
2\left(z \mp i \varepsilon_{1}\right) r^{-s}\left(G_{0}^{\sharp}-z \pm i \varepsilon_{1}\right)^{-2} r^{-s}= & -r^{-s}\left(G_{0}^{\sharp}-z \pm i \varepsilon_{1}\right)^{-1} r^{-s} \\
& +r^{-s}\left(G_{0}^{\sharp}-z \pm i \varepsilon_{1}\right)^{-1} \partial_{r} r^{-s+1} \\
& -r^{-s+1} \partial_{r}\left(G_{0}^{\sharp}-z \pm i \varepsilon_{1}\right)^{-1} r^{-s} \\
& +r^{-s}\left(G_{0}^{\sharp}-z \pm i \varepsilon_{1}\right)^{-1} \mathcal{A}\left(G_{0}^{\sharp}-z \pm i \varepsilon_{1}\right)^{-1} r^{-s} .
\end{aligned}
$$

In view of the assumptions (1.15) and (1.16), we have

$$
\mathcal{B}:=r^{\delta} \mathcal{A}\left(G_{0}^{\sharp}-i\right)^{-1} \in \mathcal{L}\left(H_{0}^{\sharp}\right) .
$$

We can write

$$
r^{-s}\left(G_{0}^{\sharp}-z \pm i \varepsilon_{1}\right)^{-1} \mathcal{A}\left(G_{0}^{\sharp}-z \pm i \varepsilon_{1}\right)^{-1} r^{-s}=r^{-s}\left(G_{0}^{\sharp}-z \pm i \varepsilon_{1}\right)^{-1} r^{-\delta / 2}
$$

$$
\begin{aligned}
\times & \mathcal{B}\left(r^{-\delta / 2-s}-\left[\partial_{r}^{2}, r^{-\delta / 2}\right]\left(G_{0}^{\sharp}-z \pm i \varepsilon_{1}\right)^{-1} r^{-s}\right. \\
& \left.+\left(z \mp i \varepsilon_{1}-i\right) r^{-\delta / 2}\left(G_{0}^{\sharp}-z \pm i \varepsilon_{1}\right)^{-1} r^{-s}\right) .
\end{aligned}
$$


Let $\mathcal{D}_{r}=-i z^{-1 / 2} \partial_{r}$. We need the following estimates (for $s>\frac{1}{2}, j=0,1$ ):

$$
\begin{aligned}
\left\|r^{-s} \mathcal{D}_{r}^{j}\left(G_{0}^{\sharp}-z \pm i \varepsilon_{1}\right)^{-1} r^{-s}\right\| & \leq C z^{-1 / 2}, \\
\left\|\mathcal{D}_{r}^{j}\left(G_{0}^{\sharp}-z \pm i \varepsilon_{1}\right)^{-1} r^{-s}\right\| & \leq C z^{-1 / 2} \varepsilon^{-1 / 2},
\end{aligned}
$$

for $z \geq C_{0}^{\prime}$, with some constants $C, C_{0}^{\prime}>0$ independent of $z$ and $\varepsilon$. Here and below $\|\cdot\|$ denotes the norm in $\mathcal{L}\left(H_{0}^{\sharp}\right)$. For $j=0,(2.20)$ follows from (1.5), while (2.21) is proved in [2] (see Lemma 3.1). For $j=1$ these estimates follow easily from the case $j=0$. Indeed, given $u \in D\left(G_{0}^{\sharp}\right)$, for $z \gg 1$ we have

$$
\begin{aligned}
\left\|r^{-s} \partial_{r} u\right\|_{H_{0}^{\sharp}}^{2} & \leq\left\|\partial_{r}\left(r^{-s} u\right)\right\|_{H_{0}^{\sharp}}^{2}+C\left\|r^{-s} u\right\|_{H_{0}^{\sharp}}^{2} \\
& \leq \operatorname{Re}\left\langle\left(G_{0}^{\sharp}-z \pm \varepsilon_{1}\right) r^{-s} u, r^{-s} u\right\rangle_{H_{0}^{\sharp}}+O(z)\left\|r^{-s} u\right\|_{H_{0}^{\sharp}}^{2} \\
& \leq \frac{1}{z}\left\|\left(G_{0}^{\sharp}-z \pm \varepsilon_{1}\right)\left(r^{-s} u\right)\right\|_{H_{0}^{\sharp}}^{2}+O(z)\left\|r^{-s} u\right\|_{H_{0}^{\sharp}}^{2} \\
& \leq \frac{1}{z}\left\|r^{-s}\left(G_{0}^{\sharp}-z \pm \varepsilon_{1}\right) u\right\|_{H_{0}^{\sharp}}^{2}+\frac{1}{z}\left\|\left[\partial_{r}^{2}, r^{-s}\right] u\right\|_{H_{0}^{\sharp}}^{2}+O(z)\left\|r^{-s} u\right\|_{H_{0}^{\sharp}}^{2} \\
& \leq \frac{1}{z}\left\|r^{-s}\left(G_{0}^{\sharp}-z \pm \varepsilon_{1}\right) u\right\|_{H_{0}^{\sharp}}^{2}+O\left(z^{-1}\right)\left\|r^{-s} \partial_{r} u\right\|_{H_{0}^{\sharp}}^{2}+O(z)\left\|r^{-s} u\right\|_{H_{0}^{\sharp}}^{2},
\end{aligned}
$$

which yields

$$
\left\|r^{-s} \partial_{r} u\right\|_{H_{0}^{\sharp}} \leq O\left(z^{-1 / 2}\right)\left\|r^{-s}\left(G_{0}^{\sharp}-z t \varepsilon_{1}\right) u\right\|_{H_{0}^{\sharp}}+O\left(z^{1 / 2}\right)\left\|r^{-s} u\right\|_{H_{0}^{\sharp}} .
$$

Clearly, this implies the desired estimates.

Given a parameter $A>1$, denote by $\chi(r \leq A)$ (resp. $\chi(r \geq A)$ ) the characteristic function of the set $\{r \leq A\}$ (resp. $\{r \geq A\}$ ). Given any $s>\frac{1}{2}, s_{1}>\frac{1}{2}$, in view of $(2.20)$ and (2.21), we have

$$
\begin{aligned}
\left\|r^{-\delta / 2} \mathcal{D}_{r}^{j}\left(G_{0}^{\sharp}-z \pm i \varepsilon_{1}\right)^{-1} r^{-s}\right\| \leq & \left\|r^{-\delta / 2} \chi(r \leq A) \mathcal{D}_{r}^{j}\left(G_{0}^{\sharp}-z \pm i \varepsilon_{1}\right)^{-1} r^{-s}\right\| \\
& +\left\|r^{-\delta / 2} \chi(r \geq A) \mathcal{D}_{r}^{j}\left(G_{0}^{\sharp}-z \pm i \varepsilon_{1}\right)^{-1} r^{-s}\right\| \\
\leq & A^{s_{1}-\delta / 2}\left\|r^{-s_{1}} \mathcal{D}_{r}^{j}\left(G_{0}^{\sharp}-z \pm i \varepsilon_{1}\right)^{-1} r^{-s}\right\| \\
& +A^{-\delta / 2}\left\|\mathcal{D}_{r}^{j}\left(G_{0}^{\sharp}-z \pm i \varepsilon_{1}\right)^{-1} r^{-s}\right\| \\
\leq & O\left(z^{-1 / 2}\right)\left(A^{s_{1}-\delta / 2}+A^{-\delta / 2} \varepsilon^{-1 / 2}\right) \\
= & O\left(z^{-1 / 2} \varepsilon^{-1 / 2+\delta / 4 s_{1}}\right),
\end{aligned}
$$

if we choose $A=\varepsilon^{-1 / 2 s_{1}}$. Taking into account that, for $s^{\prime}, s^{\prime \prime}>0$,

$$
r^{-s^{\prime}}\left(G_{0}^{\sharp}-z \pm i \varepsilon_{1}\right)^{-1} \mathcal{D}_{r} r^{-s^{\prime \prime}}=\left(r^{-s^{\prime \prime}} \mathcal{D}_{r}\left(G_{0}^{\sharp}-z \mp i \varepsilon_{1}\right)^{-1} r^{-s^{\prime}}\right)^{*}
$$

it is easy to see that (1.10) with $j=0, m=0, s=1+\frac{1}{2} \delta$ and $\mu=\delta / 2 s_{1}$, for all $s_{1}>\frac{1}{2}$ follows from (2.17) $-(2.21)$ and (2.23). The estimate (1.10) with $j=1$ follows easily from (1.10) with $j=0$ and the observation that we have an analogue of (2.22) with $G_{0}^{\sharp}$ and $H_{0}^{\sharp}$ replaced by $G_{0}$ and $H_{0}$, respectively, and $\left\|r^{-s} \nabla_{g} u\right\|_{H_{0}}$ in the left-hand side, for $u \in D\left(G_{0}\right)$. 


\section{Uniform energy estimates}

In this section we will derive (1.11) from (1.13) and (1.14). We will first prove the following result.

Proposition 3.1. (a) The estimate (1.6) implies, for $s>\frac{1}{2}$,

$$
\int_{0}^{\infty} E_{s}(\tau) d \tau \leq C \widetilde{E}_{-s}(0)
$$

(b) The estimates (1.13) and (1.14) imply, for $t \geq 1$,

$$
\int_{t}^{\infty} E_{s}(\tau) d \tau \leq C t^{-2 m-2 \mu} \widetilde{E}_{-s}(0)
$$

Proof. Choose a real-valued function $\phi(t) \in C^{\infty}(\mathbf{R}), \phi \geq 0, \phi(t)=0$ for $t \leq \frac{1}{3}$ and $\phi(t)=1$ for $t \geq \frac{1}{2}$. Let $u$ be the solution of the equation (1.7). We have

$$
\left(\partial_{t}^{2}+\Delta_{g}\right) \phi u=\left(\phi^{\prime \prime}+2 \phi^{\prime} \partial_{t}\right) u=: v(t)
$$

By Duhamel's formula,

$$
\phi u(t)=\int_{0}^{t} \frac{\sin ((t-\tau) \sqrt{G})}{\sqrt{G}} v(\tau) d \tau .
$$

On the other hand, we have the formula

$$
\left(G-(\lambda-i \varepsilon)^{2}\right)^{-1}=\int_{0}^{\infty} e^{-i t(\lambda-i \varepsilon)} \frac{\sin (t \sqrt{G})}{\sqrt{G}} d t, \quad \varepsilon>0 .
$$

It follows from (3.3) and (3.4) that the Fourier transform of $\phi u$ satisfies the identity

$$
\widehat{\phi u}(\lambda-i \varepsilon)=\left(G-(\lambda-i \varepsilon)^{2}\right)^{-1} \varphi_{1}(G) \hat{v}(\lambda-i \varepsilon),
$$

where $\varphi_{1} \in C^{\infty}(\mathbf{R}), \varphi_{1}=1$ in a neighbourhood of $\operatorname{supp} \varphi$ and $\varphi_{1}=0$ in a neighbourhood of $\left(-\infty, A_{0}\right]$. Hence, for every $\varepsilon>0$, we have

$$
\begin{aligned}
\chi_{s} \widehat{\partial_{t}(\phi u)}(\lambda-i \varepsilon) & =\chi_{s}(\lambda-i \varepsilon)\left(G-(\lambda-i \varepsilon)^{2}\right)^{-1} \varphi_{1}(G) \chi_{s} \chi_{-s} \hat{v}(\lambda-i \varepsilon) \\
\chi_{s} \nabla_{g} \widehat{\phi u}(\lambda-i \varepsilon) & =\chi_{s} \nabla_{g}\left(G-(\lambda-i \varepsilon)^{2}\right)^{-1} \varphi_{1}(G) \chi_{s} \chi_{-s} \hat{v}(\lambda-i \varepsilon)
\end{aligned}
$$

We now need the following estimate. 
Lemma 3.2. There exists a constant $C>0$ so that

$$
\left\|\chi_{-s} v(t)\right\|_{H}^{2} \leq C \widetilde{E}_{-s}(0)
$$

Proof. Let us first see that for every $s \geq 0$ there exists $a_{s} \geq 0$ so that, for all $t \in \mathbf{R}$

$$
\chi_{-s} \cos (t \sqrt{G}) \chi_{s}, \chi_{-s} \sin (t \sqrt{G}) \chi_{s}=O\left((|t|+1)^{a_{s}}\right): H \rightarrow H .
$$

Clearly, it suffices to prove (3.9) for $t>0$. By Duhamel's formula we have (3.10)

$$
\chi_{-s} \cos (t \sqrt{G}) \chi_{s}=\cos (t \sqrt{G})+\int_{0}^{t} \frac{\sin ((t-\tau) \sqrt{G})}{\sqrt{G}}\left[\Delta_{g}, \chi_{-s}\right] \cos (\tau \sqrt{G}) \chi_{s} d \tau
$$

and similarly for $\sin (t \sqrt{G})$. We have

$$
p^{1 / 2}\left[\Delta_{g}, \chi_{-s}\right] p^{-1 / 2} f=\left[-\partial_{r}^{2}, \chi_{-s}\right] f=\frac{\partial^{2} \chi_{-s}}{\partial r^{2}} f-2 \partial_{r}\left(\frac{\partial \chi_{-s}}{\partial r} f\right) .
$$

Hence, by (3.10), for every $\alpha>0$,

$$
\begin{aligned}
\left\|\chi_{-s} \cos (t \sqrt{G}) \chi_{s}\right\| \leq & 1+C \int_{0}^{t}(t-\tau)\left\|\chi_{-s+2} \cos (\tau \sqrt{G}) \chi_{s}\right\| d \tau \\
& +(1+\alpha t) \int_{0}^{t}\left\|(G+\alpha)^{-1 / 2} \tilde{\partial}_{r} \widetilde{\chi} \frac{\partial \chi_{-s}}{\partial r} \cos (\tau \sqrt{G}) \chi_{s}\right\| d \tau
\end{aligned}
$$

where $\tilde{\partial}_{r}=p^{-1 / 2} \partial_{r} p^{1 / 2}, \tilde{\chi}=1$ on $\operatorname{supp} \partial \chi_{-s} / \partial r, \tilde{\chi}=0$ on $M \backslash X_{r_{0}+1 / 3}$ and $0 \leq \tilde{\chi} \leq 1$. Choose a function $\tilde{\eta}, \tilde{\eta}=1$ on $\operatorname{supp} \tilde{\chi}, \tilde{\eta}=0$ on $M \backslash X_{r_{0}+1 / 4}, 0 \leq \tilde{\eta} \leq 1$. In view of $(1.1)$ and (1.2), we have

$$
\begin{aligned}
\left\|\widetilde{\chi} \tilde{\partial}_{r} f\right\|_{H}^{2} & \leq\left\langle\Delta_{g}(\tilde{\eta} f), \tilde{\eta} f\right\rangle_{H}+C_{1}\|f\|_{H}^{2}=\left\|\nabla_{g}(\tilde{\eta} f)\right\|_{H}^{2}+C_{1}\|f\|_{H}^{2} \\
& \leq\left\|\nabla_{g} f\right\|_{H}^{2}+C_{2}\|f\|_{H}^{2}=\langle(G+\alpha) f, f\rangle_{H}=\left\|(G+\alpha)^{1 / 2} f\right\|_{H}^{2},
\end{aligned}
$$

with $\alpha=C_{2}$. Hence

$$
\left\|(G+\alpha)^{-1 / 2} \tilde{\partial}_{r} \tilde{\chi}\right\|=\left\|\widetilde{\chi} \tilde{\partial}_{r}(G+\alpha)^{-1 / 2}\right\| \leq 1 .
$$

Thus, we obtain

$$
\begin{aligned}
\left\|\chi_{-s} \cos (t \sqrt{G}) \chi_{s}\right\| \leq & 1+C t \int_{0}^{t}\left\|\chi_{-s+2} \cos (\tau \sqrt{G}) \chi_{s}\right\| d \tau \\
& +C(1+\alpha t) \int_{0}^{t}\left\|\chi_{-s+1} \cos (\tau \sqrt{G}) \chi_{s}\right\| d \tau
\end{aligned}
$$


Clearly, a similar estimate holds for $\sin (t \sqrt{G})$ as well. It is easy to see by induction over $s$ that (3.11) implies (3.9).

In view of (1.8), we have

$$
\begin{aligned}
\chi_{-s} v(t)= & \phi^{\prime \prime}(t) \chi_{-s} \cos (t \sqrt{G}) \varphi(G) f_{1}+\phi^{\prime \prime}(t) \chi_{-s} \sin (t \sqrt{G}) G^{-1 / 2} \varphi(G) f_{2} \\
& -2 \phi^{\prime}(t) \chi_{-s} \sin (t \sqrt{G}) G^{1 / 2} \varphi(G) f_{1}+2 \phi^{\prime}(t) \chi_{-s} \cos (t \sqrt{G}) \varphi(G) f_{2}
\end{aligned}
$$

Hence, using (3.9), we obtain

$$
\begin{aligned}
\left\|\chi_{-s} v(t)\right\|_{H} \leq & C\left\|\chi_{-s} \varphi(G) f_{1}\right\|_{H}+C\left\|\chi_{-s} G^{1 / 2} \varphi(G) f_{1}\right\|_{H} \\
& +C\left\|\chi_{-s} G^{-1 / 2} \varphi(G) f_{2}\right\|_{H}+C\left\|\chi_{-s} \varphi(G) f_{2}\right\|_{H} .
\end{aligned}
$$

Now (3.8) follows from (3.12) and Lemma 3.3 below.

Lemma 3.3. The operators $\chi_{-s} \varphi(G) \chi_{s}$ and $\chi_{-s} G^{-1 / 2} \varphi(G) \chi_{s}$ belong to $\mathcal{L}(H)$. Moreover, the following estimate holds:

$$
\left\|\chi_{-s} G^{1 / 2} \varphi(G) f\right\|_{H} \leq C\left\|\chi_{-s} \nabla_{g} f\right\|_{H}+C\left\|\chi_{-s+1} f\right\|_{H} .
$$

Proof. Introduce the function $\psi(\lambda)=\varphi\left(\lambda^{2}\right)$. We are going to take advantage of the formula

$$
\varphi(G)=\psi(\sqrt{G})=\frac{1}{2 \pi} \int_{-\infty}^{\infty} e^{i t \sqrt{G}} \hat{\psi}(t) d t,
$$

where $\hat{\psi}(t)=O_{N}\left(|t|^{-N}\right), N \geq 1$, for $|t| \gg 1$, and $\hat{\psi}(t)=O\left(|t|^{-1}\right)$ for $|t| \ll 1$. Hence

$$
\chi_{-s} \varphi(G) \chi_{s}-\varphi(G)=\frac{1}{2 \pi} \int_{-\infty}^{\infty}\left(\chi_{-s} e^{i t \sqrt{G}} \chi_{s}-e^{i t \sqrt{G}}\right) \hat{\psi}(t) d t
$$

It follows from (3.9) and (3.10) that

$$
\chi_{-s} e^{i t \sqrt{G}} \chi_{s}-e^{i t \sqrt{G}} \in \mathcal{L}(H)
$$

with norm upper bounded by $O\left(|t|^{a_{s}}\right)$ for $|t| \gg 1$, and by $O(|t|)$ for $|t| \ll 1$. Therefore, the integral in (3.15) is absolutely convergent, and we conclude that the operator $\chi_{-s} \varphi(G) \chi_{s}$ belongs to $\mathcal{L}(H)$. Clearly, the operator $\chi_{-s} \varphi(G) G^{-1 / 2} \chi_{s}$ can be treated in the same way.

Set $\psi_{1}(\lambda)=\lambda \psi(\lambda)$. We have

$$
G^{1 / 2} \varphi(G)=\psi_{1}(\sqrt{G})=\frac{1}{2 \pi} \int_{-\infty}^{\infty} e^{i t \sqrt{G}} \hat{\psi}_{1}(t) d t
$$


where $\hat{\psi}_{1}(t)=O_{N}\left(|t|^{-N}\right), N \geq 1$, for $|t| \gg 1$, and $\hat{\psi}_{1}(t)=O\left(|t|^{-2}\right)$ for $|t| \ll 1$. We can write

$$
\begin{aligned}
{\left[\chi_{-s}, G^{1 / 2} \varphi(G)\right] f=} & \frac{1}{2 \pi} \int_{|t| \geq 1}\left[\chi_{-s}, e^{i t \sqrt{G}}\right] f \hat{\psi}_{1}(t) d t \\
& +\frac{1}{2 \pi} \int_{|t| \leq 1}\left[\chi_{-s}, e^{i t \sqrt{G}}\right] f \hat{\psi}_{1}(t) d t=: I_{1}+I_{2}
\end{aligned}
$$

Writing (3.10) in the form

$$
\left[\chi_{-s}, e^{i t \sqrt{G}}\right] f=\int_{0}^{t} \frac{\sin ((t-\tau) \sqrt{G})}{\sqrt{G}}\left[\Delta_{g}, \chi_{-s}\right] e^{i \tau \sqrt{G}} f d \tau
$$

and taking into account (3.9) one easily obtains

$$
\begin{array}{ll}
\left\|\left[\chi_{-s}, e^{i t \sqrt{G}}\right] f\right\|_{H} \leq O\left(|t|^{a_{s}}\right)\left\|\chi_{-s+1} f\right\|_{H}, & |t| \geq 1, \\
\left\|\left[\chi_{-s}, e^{i t \sqrt{G}}\right] f\right\|_{H} \leq O(|t|)\left\|\chi_{-s+1} f\right\|_{H}, & |t| \leq 1 .
\end{array}
$$

It follows from (3.18) that the integral $I_{1}$ is absolutely convergent, and hence

$$
\left\|I_{1}\right\|_{H} \leq C\left\|\chi_{-s+1} f\right\|_{H}
$$

By (3.17) we have, for $|t| \leq 1$,

$$
\begin{aligned}
\left\|\left[\chi_{-s}, e^{i t \sqrt{G}}\right] f\right\|_{H} \leq & \int_{0}^{t}(t-\tau) d \tau\left(\left\|\left[\Delta_{g}, \chi_{-s}\right] f\right\|_{H}+\left\|\chi_{-s+2} f\right\|_{H}\right) \\
& +\int_{0}^{t}\left\|\chi_{-s+1}\left(e^{i \tau \sqrt{G}}-1\right) f\right\|_{H} d \tau \\
\leq & C t^{2}\left(\left\|\chi_{-s+1} \nabla_{g} f\right\|_{H}+\left\|\chi_{-s+2} f\right\|_{H}\right) \\
& +\int_{0}^{t}\left\|\left[\chi_{-s+1}, e^{i \tau \sqrt{G}}\right] f\right\|_{H} d \tau+\int_{0}^{t}\left\|\left(e^{i \tau \sqrt{G}}-1\right) \chi_{-s+1} f\right\|_{H} d \tau \\
\leq & C t^{2}\left(\left\|\chi_{-s+1} \nabla_{g} f\right\|_{H}+\left\|\chi_{-s+2} f\right\|_{H}+\left\|G^{1 / 2} \chi_{-s+1} f\right\|_{H}\right),
\end{aligned}
$$

where we have used (3.19). Hence

$$
\begin{aligned}
\left\|I_{2}\right\|_{H} & \leq C\left\|\chi_{-s+1} \nabla_{g} f\right\|_{H}+C\left\|\chi_{-s+2} f\right\|_{H}+C\left\|G^{1 / 2} \chi_{-s+1} f\right\|_{H} \\
& \leq C\left\|\chi_{-s+1} \nabla_{g} f\right\|_{H}+C\left\|\chi_{-s+2} f\right\|_{H}+C\left\|\nabla_{g} \chi_{-s+1} f\right\|_{H} \\
& \leq C\left\|\chi_{-s+1} \nabla_{g} f\right\|_{H}+C\left\|\chi_{-s+2} f\right\|_{H} .
\end{aligned}
$$


By (3.16), (3.20) and (3.21),

$$
\left\|\left[\chi_{-s}, G^{1 / 2} \varphi(G)\right] f\right\|_{H} \leq C\left\|\chi_{-s+1} \nabla_{g} f\right\|_{H}+C\left\|\chi_{-s+1} f\right\|_{H}
$$

Hence

$$
\begin{aligned}
\left\|\chi_{-s} G^{1 / 2} \varphi(G) f\right\|_{H} & \leq C\left\|G^{1 / 2} \chi_{-s} f\right\|_{H}+C\left\|\chi_{-s+1} \nabla_{g} f\right\|_{H}+C\left\|\chi_{-s+1} f\right\|_{H} \\
& \leq C\left\|\nabla_{g} \chi_{-s} f\right\|_{H}+C\left\|\chi_{-s+1} \nabla_{g} f\right\|_{H}+C\left\|\chi_{-s+1} f\right\|_{H} \\
& \leq C\left\|\chi_{-s} \nabla_{g} f\right\|_{H}+C\left\|\chi_{-s+1} f\right\|_{H} .
\end{aligned}
$$

We have now proved Lemma 3.3, and hence also Lemma 3.2 and can therefore proceed with the proof of Proposition 3.1.

Let us see that, for $\lambda \in \mathbf{R}$, we have

$$
\begin{aligned}
& \left\|\frac{d^{k}}{d \lambda^{k}} \chi_{s}(\lambda-i \varepsilon)\left(G-(\lambda-i \varepsilon)^{2}\right)^{-1} \varphi_{1}(G) \chi_{s}\right\| \\
& \quad+\left\|\frac{d^{k}}{d \lambda^{k}} \chi_{s} \nabla_{g}\left(G-(\lambda-i \varepsilon)^{2} r\right)^{-1} \varphi_{1}(G) \chi_{s}\right\| \leq C, \quad k=0,1, \ldots, m, \\
& \left\|\frac{d^{m+1}}{d \lambda^{m+1}} \chi_{s}(\lambda-i \varepsilon)\left(G-(\lambda-i \varepsilon)^{2}\right)^{-1} \varphi_{1}(G) \chi_{s}\right\| \\
& +\left\|\frac{d^{m+1}}{d \lambda^{m+1}} \chi_{s} \nabla_{g}\left(G-(\lambda-i \varepsilon)^{2}\right)^{-1} \varphi_{1}(G) \chi_{s}\right\| \leq C \varepsilon^{-1+\mu},
\end{aligned}
$$

with a constant $C>0$ independent of $\lambda$ and $\varepsilon$. For $|\lambda| \geq \sqrt{A_{0}}$, (3.23) and (3.24) follow from (1.13) and (1.14), respectively, together with the fact that the operator $\chi_{-s} \varphi_{1}(G) \chi_{s}$ is bounded on $H$. Let now $|\lambda| \leq \sqrt{A_{0}}$. For every integer $k \geq 1$, we have with some constant $\varepsilon_{0}>0(j=0,1)$

$$
\begin{aligned}
\left\|\nabla_{g}^{j}\left(G-(\lambda-i \varepsilon)^{2}\right)^{-k} \varphi_{1}(G)\right\| & \leq\left\|G^{j / 2}\left(G-(\lambda-i \varepsilon)^{2}\right)^{-k} \varphi_{1}(G)\right\| \\
& \leq \sup _{y \in \mathbf{R}}\left|y^{j / 2}\left(y-(\lambda-i \varepsilon)^{2}\right)^{-k} \varphi_{1}(y)\right| \\
& \leq \sup _{y \geq A_{0}+\varepsilon_{0}}\left|y^{j / 2}\left(y-\lambda^{2}+\varepsilon^{2}\right)^{-k}\right| \\
& \leq \text { Const, }
\end{aligned}
$$

which clearly implies (3.23) and (3.24) in this case.

By (3.6), (3.7), (3.23) and (3.24), we get

$$
\left\|\chi_{s} \widehat{\partial_{t}(\phi u)}(\lambda-i \varepsilon)\right\|_{H}+\left\|\chi_{s} \nabla_{g} \widehat{\phi u}(\lambda-i \varepsilon)\right\|_{H} \leq C\left\|\chi_{-s} \hat{v}(\lambda-i \varepsilon)\right\|_{H},
$$




$$
\begin{aligned}
& \left\|\frac{d^{m+1}}{d \lambda^{m+1}} \chi_{s} \widehat{\partial_{t}(\phi u)}(\lambda-i \varepsilon)\right\|_{H}+\left\|\frac{d^{m+1}}{d \lambda^{m+1}} \chi_{s} \nabla_{g} \widehat{\phi u}(\lambda-i \varepsilon)\right\|_{H} \\
& \leq C \varepsilon^{-1+\mu}\left\|\chi_{-s} \hat{v}(\lambda-i \varepsilon)\right\|_{H}+C \sum_{k=1}^{m+1}\left\|\chi_{-s} \widehat{t^{k} v}(\lambda-i \varepsilon)\right\|_{H}
\end{aligned}
$$

with a constant $C>0$ independent of $\lambda$ and $\varepsilon$. By Plancherel's identity we have, for every $0<\varepsilon \ll 1$,

$$
\begin{aligned}
\int_{-\infty}^{\infty}\left(\left\|\chi_{s} \partial_{t}(\phi u)\right\|_{H}^{2}\right. & \left.+\left\|\chi_{s} \nabla_{g} \phi u\right\|_{H}^{2}\right) e^{-2 \varepsilon t} d t \\
& =C_{1} \int_{-\infty}^{\infty}\left(\left\|\chi_{s} \widehat{\partial_{t}(\phi u)}(\lambda-i \varepsilon)\right\|_{H}^{2}+\left\|\chi_{s} \nabla_{g} \widehat{\phi u}(\lambda-i \varepsilon)\right\|_{H}^{2}\right) d \lambda \\
& \leq C_{2} \int_{-\infty}^{\infty}\left\|\chi_{-s} \hat{v}(\lambda-i \varepsilon)\right\|_{H}^{2} d \lambda \\
& =C_{3} \int_{-\infty}^{\infty}\left\|\chi_{-s} v(t)\right\|_{H}^{2} e^{-2 \varepsilon t} d t \\
& \leq C \widetilde{E}_{-s}(0)
\end{aligned}
$$

with a constant $C>0$ independent of $\varepsilon$, where we have used $(3.25),(3.8)$ and the fact that $\chi_{-s} v(t)$ has compact support in $t$. Hence, we can take $\varepsilon \rightarrow 0$ in $(3.27)$, which clearly implies (3.1).

To prove (3.2) we proceed similarly as above. We have, for every $T \geq 1$ and every $0<\varepsilon \ll 1$,

$$
\begin{aligned}
e^{-4 \varepsilon T} T^{2 m+2} \int_{T}^{2 T}\left(\left\|\chi_{s} \partial_{t}(\phi u)\right\|_{H}^{2}+\left\|\chi_{s} \nabla_{g} \phi u\right\|_{H}^{2}\right) d t \\
\quad \leq \int_{-\infty}^{\infty}\left(\left\|\chi_{s} t^{m+1} \partial_{t}(\phi u)\right\|_{H}^{2}+\left\|\chi_{s} t^{m+1} \nabla_{g} \phi u\right\|_{H}^{2}\right) e^{-2 \varepsilon t} d t \\
\quad=C_{1} \int_{-\infty}^{\infty}\left(\left\|\frac{d^{m+1}}{d \lambda^{m+1}} \chi_{s} \widehat{\partial_{t}(\phi u)}(\lambda-i \varepsilon)\right\|_{H}^{2}+\| \frac{d^{m+1}}{\left.d \lambda^{m+1} \chi_{s} \nabla_{g} \widehat{\phi u}(\lambda-i \varepsilon) \|_{H}^{2}\right) d \lambda}\right. \\
\leq C_{2} \varepsilon^{-2+2 \mu} \int_{-\infty}^{\infty}\left\|\chi_{-s} \hat{v}(\lambda-i \varepsilon)\right\|_{H}^{2} d \lambda+C_{2} \sum_{k=1}^{m+1} \int_{-\infty}^{\infty}\left\|\chi_{-s} \widehat{t^{k} v}(\lambda-i \varepsilon)\right\|_{H}^{2} d \lambda \\
\quad=C_{3} \varepsilon^{-2+2 \mu} \int_{-\infty}^{\infty}\left\|\chi_{-s} v(t)\right\|_{H}^{2} e^{-2 \varepsilon t} d t+C_{3} \sum_{k=1}^{m+1} \int_{-\infty}^{\infty}\left\|\chi_{-s} t^{k} v(t)\right\|_{H}^{2} e^{-2 \varepsilon t} d t \\
\leq C \varepsilon^{-2+2 \mu} \widetilde{E}_{-s}(0),
\end{aligned}
$$

with a constant $C>0$ independent of $\varepsilon$, where we have used (3.26) and (3.8). Taking 
$\varepsilon=T^{-1}$ in $(3.28)$ we get

$$
\int_{T}^{2 T}\left(\left\|\chi_{s} \partial_{t}(\phi u)\right\|_{H}^{2}+\left\|\chi_{s} \nabla_{g} \phi u\right\|_{H}^{2}\right) d t \leq C T^{-2 m-2 \mu} \widetilde{E}_{-s}(0)
$$

for all $T \geq 1$, with a constant $C>0$ independent of $T$. Hence, for every integer $j \geq 0$, we have

$$
\int_{2^{j} T}^{2^{j+1} T}\left(\left\|\chi_{s} \partial_{t}(\phi u)\right\|_{H}^{2}+\left\|\chi_{s} \nabla_{g} \phi u\right\|_{H}^{2}\right) d t \leq C 2^{-(2 m+2 \mu) j} T^{-2 m-2 \mu} \widetilde{E}_{-s}(0) .
$$

Summing up (3.30) we get (3.2).

The estimate (1.11) follows from (3.2) and the following lemma.

Lemma 3.4. For $s>\frac{1}{2}$ and $t \geq 1$, we have

$$
E_{s-1 / 2}(t) \leq C \int_{t}^{\infty} E_{s}(\tau) d \tau
$$

with a constant $C>0$ independent of $t$.

Proof. Using Green's formula we get

$$
\begin{aligned}
\frac{d E_{s-1 / 2}(t)}{d t}= & 2 \operatorname{Re} \int_{M}\left(-\Delta_{g} u(t, x) \cdot \overline{\partial_{t} u(t, x)}+\nabla_{g} u(t, x) \cdot \overline{\nabla_{g} \partial_{t} u(t, x)}\right) \chi_{2 s-1} d \operatorname{Vol}_{g} \\
= & 2 \operatorname{Re} \int_{M}\left(-\nabla_{g} u(t, x) \cdot \overline{\nabla_{g}\left(\chi_{2 s-1} \partial_{t} u(t, x)\right)}\right. \\
& \left.+\chi_{2 s-1} \nabla_{g} u(t, x) \cdot \overline{\nabla_{g} \partial_{t} u(t, x)}\right) d \operatorname{Vol}_{g} \\
= & -2 \operatorname{Re} \int_{M} \partial_{r} u(t, x) \cdot \overline{\partial_{t} u(t, x)} \frac{\partial \chi_{2 s-1}}{\partial r} d \operatorname{Vol}_{g} .
\end{aligned}
$$

Hence, for $T>t \geq 1$, we have

$$
E_{s-1 / 2}(t) \leq E_{s-1 / 2}(T)+C \int_{t}^{T} E_{s}(\tau) d \tau
$$

Therefore, to prove (3.31) it suffices to show that for every $s>0$ there exists $T_{j} \rightarrow+\infty$ such that

$$
\lim _{T_{j} \rightarrow+\infty} E_{s}\left(T_{j}\right)=0
$$

provided $\widetilde{E}_{-s-1 / 2}(0)<+\infty$. For $s>\frac{1}{2},(3.33)$ follows from $(3.1)$. Let now $0<s \leq \frac{1}{2}$ and $s_{1}>\frac{1}{2}$. Given any $A>1$, by an interpolation argument we have

$$
E_{s}(t) \leq A^{s_{1}-s} E_{s_{1}}(t)+A^{-s} E_{0}(0) \text {. }
$$

We have that for every $\varepsilon>0$ there exists $T_{\varepsilon}>0$ such that $E_{s_{1}}\left(T_{\varepsilon}\right) \leq \varepsilon$. Hence,

$$
E_{s}\left(T_{\varepsilon}\right) \leq A^{s_{1}-s_{\varepsilon}}+A^{-s} E_{0}(0)=O\left(\varepsilon^{s / s_{1}}\right),
$$

if we choose $A=\varepsilon^{-1 / s_{1}}$, which clearly implies (3.33) in this case as well. 


\section{References}

1. Cardoso, F. and Vodev, G., Uniform estimates of the resolvent of the LaplaceBeltrami operator on infinite volume Riemannian manifolds. II, Ann. $H$. Poincaré 3 (2002), 673-691.

2. Cardoso, F. and Vodev, G., High frequency resolvent estimates and energy decay of solutions to the wave equation, to appear in Canadian Math. Bull.

3. Melrose, R. B. and Sjöstrand, J., Singularities of boundary value problems. I, Comm. Pure Appl. Math. 31 (1978), 593-617.

4. Melrose, R. B. and SJÖstrand, J., Singularities of boundary value problems. II, Comm. Pure Appl. Math. 35 (1982), 129-168.

5. VAINBERG, B. R., On the short wave asymptotic behaviour of solutions of stationary problems and the asymptotic behaviour as $t \rightarrow \infty$ of solutions of nonstationary problems, Uspekhi Mat. Nauk 30:2(182) (1975), 3-55 (Russian). English transl.: Russian Math. Surveys 30:2 (1975), 1-53.

6. VainberG, B. R., Asymptotic Methods in Equations of Mathematical Physics, Moskov. Gos. Univ., Moscow, 1982 (Russian). English transl.: Gordon and Breach, New York, 1989.

7. VASY, A. and Zworski, M., Semiclassical estimates in asymptotically Euclidean scattering, Comm. Math. Phys. 212 (2000), 205-217.

8. VODEv, G., Uniform estimates of the resolvent of the Laplace-Beltrami operator on infinite volume Riemannian manifolds with cusps, Comm. Partial Differential Equations 27 (2002), 1437-1465.

\author{
Georgi Vodev \\ Université de Nantes \\ Département de Mathématiques \\ UMR $6629 \mathrm{du}$ CNRS \\ 2, rue de la Houssinière, BP 92208 \\ FR-44322 Nantes Cedex 03 \\ France \\ email: vodev@math.univ-nantes.fr
}

